\title{
PHENOTYPIC AND GENOTYPIC ANALYSIS OF BIOFILM FORMATION IN STAPHYLOCOCCUS EPIDERMIDIS ISOLATED FROM CLINICAL SAMPLES
}

\author{
M. Saifi ${ }^{1}$, H. Vatankhah ${ }^{2}$, D. Shahbazzadeh ${ }^{3}$, M.R. Pourshafie ${ }^{2}$ \\ ${ }^{1}$ Mycobacteriology, ${ }^{2}$ Microbiology, ${ }^{3}$ Biotechnology, Pasteur Institute of Iran, Tehran, Iran
}

Objectives: Staphylococcus epidermidis is the most common cause of nosocomial infection associated with the use of indwelling medical devices. The aim of this study was the investigation of biofilm production and antibiotic resistance pattern between S.epidermidis strains isolated from hospitalized and healthy persons.

Methods: Fifty and 20 isolates of $S$. epidermidis strains from hospitalized and healty persons were collected and identified, respectively. Biofilm production was examined using qualitative and quantitative techniques. Antibiotic resistance patterns were accomplished according to the CLSI standards. The presence of biofilm production genes by PCR method were investigated. Genotyping of strains by using RFLP method was performed.

Results: Eighty six percent of hospital and $10 \%$ of healty strains were biofilm positive. In quantitative method among hospitalized patients, $68 \%$ and $15 \%$ of strains were strong adherent. Antibiotic resistance percents to penicillin, erythromycin, clindamycin and cotrimoxazole in hospital strains were more than $50 \%$ wherease among healthy strains were lower. Presence of icaA, icaB, icaC, icaD, aap ,IS256 genes and ica Operon in hospital strains were $74 \%, 68 \%, 60 \%, 84 \%, 78 \%, 72 \%$ and $58 \%$ respectively. Among strains isolated from healthy individuals presence of these genes were lower. Genotyping of strains identified 2 genotype (RP62A and 97-337).

Conclusion: Biofilm production was more common among hospital strains in comparison to healthy strains. Antibiotic resistance rate in hospital strains were higher. These characteristics of S.epidermidis can be used to distinguish between invasive strains and normal flora. Genotyping results showed the dominant genotype has higher antibiotic resistance and produced more biofilm in quantitative method. 